\title{
RIGIDITY OF CLOSED METRIC MEASURE SPACES WITH NONNEGATIVE CURVATURE
}

\author{
JIA-YONG WU
}

\begin{abstract}
We show that one-dimensional circle is the only case for closed smooth metric measure spaces with nonnegative Bakry-Émery Ricci curvature whose spectrum of the weighted Laplacian has an optimal positive upper bound. This result extends the work of Hang-Wang in the manifold case (Int. Math. Res. Not. 18 (2007), Art. ID rnm064, 9pp).
\end{abstract}

\section{INTRODUCTION}

Let $(M, g)$ be an $n$-dimensional closed Riemannian manifold and $f \in C^{2}(M)$. We define a weighted Laplacian on $M$

$$
\Delta_{f}:=\Delta-\nabla f \cdot \nabla
$$

which is a self-adjoint operator with respect to the weighted measure $e^{-f} d v$ (for short $d \mu$ ), where $d v$ is the volume element induced by the metric $g$. The weighted Laplacian is very much related to the Laplacian of a suitable conformal change of the background Riemannian metric. It also naturally arises in potential theory, probability theory and harmonic analysis on complete Riemannian manifolds. Here, the triple $\left(M, g, e^{-f} d v\right)$ is customarily called a smooth metric measure space.

On the smooth metric measure space $\left(M, g, e^{-f} d v\right)$, Bakry-Émery [4] (see also [21, 23]) introduced the Bakry-Émery Ricci curvature

$$
\operatorname{Ric}_{f}:=\operatorname{Ric}+\operatorname{Hess}(f),
$$

where Ric denotes the Ricci curvature of the manifold and Hess denotes the Hessian with respect to the Riemannian metric. A remarkable feather of $R i c_{f}$ is that this tensor relates to the weighted Laplacian via the following Bochner formula

$$
\Delta_{f}|\nabla u|^{2}=2|\operatorname{Hess}(u)|^{2}+2\left\langle\nabla u, \nabla \Delta_{f} u\right\rangle+2 \operatorname{Ric}_{f}(\nabla u, \nabla u) .
$$

Moreover, Bakry-Émery Ricci curvature is related to the gradient Ricci soliton:

$$
\operatorname{Ric}_{f}=\lambda g,
$$

where $\lambda$ is some real constant. The gradient Ricci soliton is called expanding, steady and shrinking, accordingly when $\lambda<0, \lambda=0$ and $\lambda>0$. As we all know, The Ricci soliton plays an important role in the theory of the Ricci flow [7]. It is a

Date: Published in Kodai Math. J. 39 (2016) 489-499. Originally submitted to another journal on Feb. 16, 2013.

2010 Mathematics Subject Classification. Primary 53C24; Secondary 53C21, 35P15.

Key words and phrases. Bakry-Émery Ricci curvature, weighted Laplacian, eigenvalue, rigidity. This work was partially supported by the NSFC $(11101267,11271132)$ and the Innovation Program of Shanghai Municipal Education Commission (13YZ087). 
special solution of the Ricci flow and often arises from the blow up analysis of the singularities of the Ricci flow [14.

By the variational characterization, the first nontrivial eigenvalue of the weighted Laplacian on closed metric measure space $\left(M, g, e^{-f} d v\right)$ with respect to the weighted measure $d \mu$ is defined by

$$
\lambda_{1}:=\inf _{\phi \neq 0}\left\{\int_{M}(\nabla \phi, \nabla \phi) d \mu,: \int_{M}|\phi|^{2} d \mu=1, \int_{M} \phi d \mu=1, \phi \in C^{\infty}(M)\right\} .
$$

The above infimum can be achieved by some smooth eigenfunction $\phi$. Meanwhile the eigenfunction $\phi$ satisfies the Euler-Lagrange equation

$$
\Delta_{f} \phi=-\lambda_{1} \phi .
$$

We easily see that if potential function $f$ is constant, then $R i c_{f}$ recovers the ordinary Ricci curvature and the above formulas all reduce to the classical case.

Many interesting rigid results involving Bakry-Émery Ricci curvature have been studied in large part due to their similar properties between Bakry-Émery curvature and Ricci curvature. We refer the readers to [5], 8], [11, [16, [17, [18, [22, [23, 29, 30, 31] and reference therein. In particular, Munteanu and Wang [24, 25, Su and Zhang [28, and the author [32] proved many interesting splitting results on complete noncompact metric measure spaces under some assumptions on BakryÉmery Ricci curvature. Recently, various Liouville-type theorems on smooth metric measure spaces were obtained, see for example [27] and [33]-37].

In this paper, we continue to discuss a rigid result on the closed smooth metric measure space rather than the complete noncompact case. Before introducing our result, we first recall some well-known eigenvalue estimates on closed smooth manifolds with nonnegative Ricci curvature. As we all know, Li and Yau [20] applied gradient estimate technique to give a lower bound of the first eigenvalue of the Laplace operator on a closed manifold with nonnegative Ricci curvature:

$$
\lambda_{1} \geq \frac{\pi^{2}}{2 d^{2}}
$$

where $d$ is the diameter of the manifold. Later, Zhong and Yang [38 improved this result to

$$
\lambda_{1} \geq \frac{\pi^{2}}{d^{2}} .
$$

Recently, there exist some alternate proofs of this result in [1, 2] and 26] . We also see that the above estimate is optimal as equality holds on $S^{1}$. Moreover, Hang and Wang [15] proved that $S^{1}$ is the only case for the case $\lambda_{1}=\pi^{2} / d^{2}$. Their proof relies on a strong maximum principle and a careful geometrical analysis, which is not only to simply analyze the proof course of the Zhang-Yang's inequality becoming the equality. On the other hand, Zhong-Yang's result was extended by Chen and Wang [9, 10] via probabilistic approach, and further generalized by Bakry and Qian [6] to the smooth metric measure spaces. In particular, they proved that

Theorem A. Let $\left(M, g, e^{-f} d v\right)$ be a closed smooth metric measure space with nonnegative Bakry-Émery Ricci curvature. Then

$$
\lambda_{1} \geq \frac{\pi^{2}}{d^{2}}
$$

where $d$ is the diameter of the manifold $M$. 
We remark that Theorem $\mathrm{A}$ has been generalized by B. Andrews and L. Ni [3], and A. Futaki and Y. Sano [13, and further improved by A. Futaki, H.-Z. Li and X.-D. Li [12] based on the arguments of Chen and Wang [9, 10].

Motivated by the Hang-Wang's result [15, we may ask if there exists a HangWang type rigid result in closed smooth metric measure spaces. That is to say whether or not $S^{1}$ is the only example for the case $\lambda_{1}=\pi^{2} / d^{2}$ in Theorem A? The purpose of this short note is to give an affirmative answer. Our main result is

Theorem 1.1. Let $\left(M, g, e^{-f} d v\right)$ be a closed smooth metric measure space with nonnegative Bakry-Émery Ricci curvature. Assume that the first nontrivial eigenvalue of the weighted Laplacian satisfies

$$
\lambda_{1}=\frac{\pi^{2}}{d^{2}},
$$

where $d$ is the diameter of the manifold $M$. Then $M$ is isometric to the circle of radius $\frac{d}{\pi}$ and $f$ is constant.

The main arguments to prove Theorem 1.1 comes from Hang-Wang [15], where the gradient estimate, the maximum principle and some analysis trick are explored. In our case, the proof not only depends on Hang-Wang's arguments [15], but also relies on the weighted gradient estimate and the weighted Bochner formula. If $f$ is constant, then Theorem 1.1 returns to Hang-Wang's result.

Remark 1.2. Recently, S. Lakzian [19] extended Hang-Wang's rigidity result to a general setting of metric measure spaces satisfying $R C D(0, N)$ curvature-dimension conditions. If manifold $M$ is complete noncompact, Munteanu and Wang 24] established a sharp upper bound of the first nonzero eigenvalue of the weighted Laplacian in terms of the linear growth rate of $f$. They also proved that if equality holds on the eigenvalue upper estimate and $M$ is not connected at infinity, then $M$ must be a cylinder.

By modifying the proof of Theorem 1.1, we also have a similar result for the first nonzero eigenvalue of the weighted Laplacian with respect to the Neumann boundary condition of a smooth metric measure space.

Theorem 1.3. Let $\left(M, g, e^{-f} d v\right)$ be a compact smooth metric measure space with nonnegative Bakry-Émery Ricci curvature and nonempty convex boundary. Then the first nontrivial eigenvalue of the weighted Laplacian with respect to the Neumann boundary condition satisfies

$$
\mu_{1} \geq \frac{\pi^{2}}{d^{2}}
$$

where $d$ is the diameter of the manifold $M$. Moreover if the above inequality becomes equality, then $M$ is isometric to a line segment and $f$ is constant.

The rest of this paper is organized as follows. In Section 2, we first recall the proof of Theorem $\mathrm{A}$ and then give an important lemma (see Lemma 2.2). In Section 3. we apply Lemma 2.2 and the strong maximum principle to prove Theorem 1.1 .

Acknowledgement. The author would like to thank the referee for pointing out mini errors and making valuable suggestions for the earlier version of this paper. 


\section{A KEY LEMMA}

In this section, we first recall the proof of Theorem $\mathrm{A}$, which nearly follows the proofs of Li-Yau [20] and Zhong-Yang [38. Here we sketch the proof for the reader's convenience. Let $\left(M, g, e^{-f} d v\right)$ be a closed smooth metric measure space. Let $\phi$ be the first eigenfunction of the weighted Laplacian. By multiplying with a constant it is possible to arrange that

$$
a-1=\min _{M} \phi, \quad a+1=\max _{M} \phi,
$$

where $0 \leq a(\phi)<1$ is the median of $\phi$. Letting $u=\phi-a$, then

$$
\Delta_{f} u=-\lambda_{1}(u+a) .
$$

Following the arguments of [20] and [38, we can establish the following gradient estimate of the function $u$.

Proposition 2.1. Let $\left(M, g, e^{-f} d v\right)$ be a closed smooth metric measure space with nonnegative Bakry-Émery Ricci curvature. Then

$$
|\nabla u|^{2} \leq \lambda_{1}\left(1-u^{2}\right)+2 a \lambda_{1} z(u),
$$

where $u=\phi-a$ and

$$
z(u)=\frac{2}{\pi}\left(\arcsin u+u \sqrt{1-u^{2}}\right)-u, \quad u \in[-1,1] .
$$

It is clear that $z(u)$ is continuous on $[-1,1]$ and $z(-u)=-z(u)$. From Proposition 2.1. we can deduce $\lambda_{1} \geq \pi^{2} / d^{2}$ as follows. Let $x_{1}, x_{2} \in M$, such that $u\left(x_{1}\right)$ is the maximizing point and $u\left(x_{2}\right)$ is the minimizing point. Take a minimal geodesic $\gamma$ from $x_{2}$ to $x_{1}$ with length at most $d$. Integrating the estimate (2) along this segment with respect to arclength and using oddness,

$$
\begin{aligned}
d \lambda_{1}^{1 / 2} \geq \lambda_{1}^{1 / 2} \int_{\gamma} d s & \geq \int_{\gamma} \frac{|\nabla u| d s}{\sqrt{1-u^{2}+2 a z(u)}} \\
& \geq \int_{0}^{1}\left(\frac{1}{\sqrt{1-u^{2}+2 a z}}+\frac{1}{\sqrt{1-u^{2}-2 a z}}\right) d u \\
& \geq \int_{0}^{1} \frac{1}{\sqrt{1-u^{2}}}\left(2+\frac{3 a^{2} z^{2}}{1-u^{2}}\right) d u \\
& \geq \pi+3 a^{2}\left(\int_{0}^{1} \frac{z d u}{\sqrt{1-u^{2}}}\right)^{2} \\
& =\pi+\frac{3 a^{2}}{\pi^{2}}\left(\frac{\pi}{2}-1\right)^{4} .
\end{aligned}
$$

Hence $\lambda_{1} \geq \pi^{2} / d^{2}$ and the inequality is strict unless $a=0$ (i.e. $\min _{M} u=-1$ ).

From the above proof, we easily see that on $S^{1}$, the above inequalities all become equality. Naturally, we ask if $S^{1}$ is the only case for the equality case. The answer is YES! In the rest of this note, we will explain this fact.

At first, we derive a differential inequality on the dense open set which consists of all regular points of the eigenfunction.

Lemma 2.2. Let $\left(M^{n}, g, e^{-f} d v\right)$ be a closed smooth metric measure space. Let $u$ be a nonzero smooth function on this measure space such that

$$
\Delta_{f} u=-\lambda u .
$$


Then on $\Omega=\{\nabla u \neq 0\}$,

$$
\Delta_{f} \psi-\frac{\nabla \psi \cdot \nabla\left(\psi-2 \lambda u^{2}\right)}{2|\nabla u|^{2}} \geq 2 \operatorname{Ric}_{f}(\nabla u, \nabla u),
$$

where $\psi:=|\nabla u|^{2}+\lambda u^{2}$.

Proof of Lemma 2.2. The proof of this result follows from that of Lemma 1 in [15] with little modification, but is included for completeness. Following the computation method of [20], let $e_{1}, e_{2}, \ldots, e_{n}$ be a local orthonormal frame field on $M^{n}$. We adopt the notation that subscripts in $i, j$, and $k$, with $1 \leq i, j, k \leq n$, mean covariant differentiations in the $e_{i}, e_{j}$ and $e_{k}$, directions respectively.

Differentiating $\psi$ in the direction of $e_{i}$, we have

$$
\psi_{i}=2 \sum_{j} u_{j} u_{i j}+2 \lambda u u_{i}
$$

and so

$$
\left|\frac{1}{2} \nabla \psi-\lambda u \nabla u\right|^{2}=\sum_{i}\left(\sum_{j} u_{j} u_{i j}\right)^{2} \leq\left|\nabla^{2} u\right|^{2} \cdot|\nabla u|^{2},
$$

where the summation convention is adopted on repeated indices. This implies

$$
\frac{1}{4}|\nabla \psi|^{2}-\lambda u \nabla u \cdot \nabla \psi \leq|\nabla u|^{2}\left(\left|\nabla^{2} u\right|^{2}-\lambda^{2} u^{2}\right) .
$$

Therefore on $\Omega=\{\nabla u \neq 0\}$, we have

$$
\left|\nabla^{2} u\right|^{2}-\lambda^{2} u^{2} \geq \frac{|\nabla \psi|^{2}-4 \lambda u \nabla u \cdot \nabla \psi}{4|\nabla u|^{2}}=\frac{\nabla\left(\psi-2 \lambda u^{2}\right) \cdot \nabla \psi}{4|\nabla u|^{2}} .
$$

On the other hand, using the Bochner formula (10), we conclude that

$$
\begin{aligned}
\frac{1}{2} \Delta_{f} \psi & =\left|\nabla^{2} u\right|^{2}+\nabla u \cdot \nabla \Delta_{f} u+R i c_{f}(\nabla u, \nabla u)+\lambda|\nabla u|^{2}+\lambda u \Delta_{f} u \\
& =\left|\nabla^{2} u\right|^{2}-\lambda^{2} u^{2}+\operatorname{Ric}_{f}(\nabla u, \nabla u),
\end{aligned}
$$

where we used $\Delta_{f} u=-\lambda u$. Combining this with (4) yields (3).

\section{Proof of Theorem 1.1}

In this section we will prove Theorem 1.1. Since the idea of proof comes from Hang-Wang [15, we only provide main steps and omit tedious discussions.

Proof of Theorem 1.1. Assume that $\lambda_{1}=\pi^{2} / d^{2}$. From the proof of Proposition 2.1 in Section 2, we easily get $a=0$, and hence

$$
\min _{M} u=-1 \quad \text { and } \quad \max _{M} u=1,
$$

where $u=\phi$ is a first eigenfunction. By scaling the metric, we can assume $d=\pi$. So $\lambda_{1}=1$. Let $\psi=|\nabla u|^{2}+u^{2}$. By Lemma 2.2, on $\Omega=\{\nabla u \neq 0\}$, we have

$$
\Delta_{f} \psi-\frac{\nabla \psi \cdot \nabla\left(\psi-2 \lambda u^{2}\right)}{2|\nabla u|^{2}} \geq 0 .
$$

Using the maximum principle, we conclude that

$$
\psi:=|\nabla u|^{2}+u^{2} \leq \max _{\{\nabla u=0\}}\left(|\nabla u|^{2}+u^{2}\right)=1,
$$

since $\psi$ can not attain the maximum value at the point of $\Omega=\{\nabla u \neq 0\}$. 
Take two points $p_{0}$ and $p_{1}$ such that

$$
u\left(p_{0}\right)=-1 \text { and } u\left(p_{1}\right)=1,
$$

and let $\gamma:[0, l] \rightarrow M$ be a unit speed minimizing geodesic from $p_{0}$ to $p_{1}$. We define a function $y(t)=u(\gamma(t))$. Then

$$
\left|y^{\prime}(t)\right|=\left|\nabla u(\gamma(t)) \cdot \gamma^{\prime}(t)\right| \leq|\nabla u(\gamma(t))| \leq \sqrt{1-y^{2}(t)} .
$$

Hence

$$
\pi \geq l \geq \int_{\left\{0 \leq t \leq l, y^{\prime}(t)>0\right\}} d t \geq \int_{0}^{l} \frac{y^{\prime}(t) d t}{\sqrt{1-y^{2}(t)}}=\int_{-1}^{1} \frac{d x}{\sqrt{1-x^{2}}}=\pi .
$$

Therefore

$$
l=\pi \quad \text { and } \quad y^{\prime}(t)>0
$$

for almost every $t \in(0, \pi)$. Hence $y(t)$ is strictly increasing on $[0, \pi]$. Moreover, we also have

$$
\int_{0}^{\pi} \frac{y^{\prime}(t) d t}{\sqrt{1-y^{2}(t)}}=\pi
$$

which implies that $y^{\prime}(t)=\sqrt{1-y^{2}(t)}$ for all $t \in[0, \pi]$. Since $y(0)=-1$ and $y^{\prime}(0)=0$, then

$$
y(t)=u(\gamma(t))=-\cos t
$$

for $t \in[0, \pi]$. It follows that

$$
\left(\nabla^{2} u\right)\left(\gamma^{\prime}(0), \gamma^{\prime}(0)\right)=1
$$

Since $\Delta_{f} u\left(p_{0}\right)=-\lambda_{1} u(\gamma(0))=1,(\nabla f \cdot \nabla u)\left(p_{0}\right)=0$ and $\left(\nabla^{2} u\right)_{p_{0}} \geq 0$, we conclude that $\Delta u\left(p_{0}\right)=1$ and hence we must have

$$
\left(\nabla^{2} u\right)_{p_{0}}=\lambda_{\gamma^{\prime}(0)} \otimes \lambda_{\gamma^{\prime}(0)},
$$

where for any tangent vector $X, \lambda_{X}$ is the dual cotangent vector given by $\lambda_{X}(Y)=$ $\langle X, Y\rangle$ for any tangent vector $Y$.

Next, similar to the Hang-Wang's argument [15], we get

Proposition 3.1. The set $\{u= \pm 1\}$ has at most four points.

Proof. We only discuss the case $\{u=1\}$ since the case $\{u=-1\}$ is similar. For any point $p$ with $u(p)=1$, we choose a minimizing geodesic $\gamma_{p}:\left[0, l_{p}\right] \rightarrow M$ from $p_{0}$ to $p$. Then the same argument as before shows that

$$
l_{p}=\pi \quad \text { and } \quad\left(\nabla^{2} u\right)_{p_{0}}=\lambda_{\gamma_{p}^{\prime}(0)} \otimes \lambda_{\gamma_{p}^{\prime}(0)},
$$

which implies $\gamma_{p}^{\prime}(0)= \pm \gamma^{\prime}(0)$. Hence $p=\exp \left(\pi \gamma_{p}^{\prime}(0)\right)$ has at most two choices.

In the next step, to finish the proof of Theorem 1.1, we only need to claim that the dimension of $M$ must be one. Argue by contradiction. If the claim is not true, then we assume that $\operatorname{dim} M \geq 2$. If we let

$$
M^{*}=M \backslash\{u= \pm 1\},
$$

then $M^{*}$ is still connected. In the following we want to show $|\nabla u|^{2}+u^{2}=1$ on $M^{*}$. In fact, we consider

$$
E=\left\{p \in M^{*}:|\nabla u(p)|^{2}+u^{2}(p)=1\right\} .
$$


Clearly, $E$ is closed. On the other hand, if $p \in E \subset \Omega$, by (5) and the strong maximum principle, we have

$$
|\nabla u|^{2}+u^{2} \equiv 1
$$

near $p$. Hence $E$ must be either an empty set or $M^{*}$. Since for any $t \in(0, \pi)$,

$$
|\nabla u(\gamma(t))|^{2}+u^{2}(\gamma(t)) \geq \cos ^{2} t+\sin ^{2} t=1,
$$

we see $E$ is nonempty and therefore $E=M^{*}$. Now we define $X=\frac{\nabla u}{|\nabla u|}$ on $M^{*}$. Since $|\nabla u|^{2}+u^{2} \equiv 1$, differentiating it yields

$$
\nabla^{2} u(X, X)=-u \text {. }
$$

We also notice that the proof of Lemma 2.2 easily implies that

$$
\left|\nabla^{2} u\right|^{2}=u^{2}
$$

on $M^{*}$, since $\psi=|\nabla u|^{2}+u^{2} \equiv 1$. Combining the above two equalities, we have

$$
\nabla^{2} u=-u \lambda_{X} \otimes \lambda_{X}
$$

Direct calculation shows that $\nabla_{X} X=0$, and hence all integral curves of $X$ are geodesics. Let $\Sigma=\{u=0\}$. Since $|\nabla u|=1$ on $\Sigma$, we see that $\Sigma$ is a hypersurface, which may have more than one components. For any $p \in \Sigma$, let $\alpha_{p}$ be the maximal integral curve of $-X$ with $\alpha_{p}(0)=p$. Then $\alpha_{p}$ is a unit speed geodesic. Letting $y_{p}(t)=u\left(\alpha_{p}(t)\right)$, we know that

$$
y_{p}(0)=0 \quad \text { and } \quad y_{p}^{\prime}(t)=-\sqrt{1-y_{p}^{2}(t)}
$$

It gives that

$$
y_{p}(t)=-\sin t \quad \text { for } \quad t \in[0, \pi / 2)
$$

On the other hand, $\alpha_{p}$ is a geodesic on $M$, defined on $[0, \infty)$. We have

$$
u\left(\alpha_{p}(t)\right)=-\sin t
$$

for $t \in[0, \pi / 2]$. In particular, $u\left(\alpha_{p}(\pi / 2)\right)=-1$. The same argument as before shows

$$
\left(\nabla^{2} u\right)_{\alpha_{p}\left(\frac{\pi}{2}\right)}=\lambda_{\alpha_{p}^{\prime}\left(\frac{\pi}{2}\right)} \otimes \lambda_{\alpha_{p}^{\prime}\left(\frac{\pi}{2}\right)} .
$$

Here $p=\exp _{\alpha_{p}\left(\frac{\pi}{2}\right)}\left(-\frac{\pi}{2} \alpha_{p}^{\prime}\left(\frac{\pi}{2}\right)\right)$. Since there are at most two points in the set $\{u=-1\}$, we may find point $q$ satisfying $u(q)=-1$ and infinitely many $p \in \Sigma$ such that $\alpha_{p}\left(\frac{\pi}{2}\right)=q$. This clearly leads to a contradiction since $\alpha_{p}^{\prime}\left(\frac{\pi}{2}\right)$ has at most two choices. Therefore the dimension of $M$ must be one. At this time, we easily see that $\operatorname{Ric}(M)=0$ and $\operatorname{Hess}(f) \geq 0$ on $S^{1}$. Hence $f^{\prime \prime}(t)=0$ on $S^{1}$, and $f$ is constant.

\section{REFERENCES}

1. B. Andrews and J. Clutterbuck, Proof of the fundamental gap conjecture, J. Amer. Math. Soc. 24 (2011), 899-916.

2. B. Andrews and J. Clutterbuck, Sharp modulus of continuity for parabolic equations on manifolds and lower bounds for the first eigenvalues, Analysis and PDE, 6 (2013), 1013-1024.

3. B. Andrews And L. Ni, Eigenvalue comparison on Bakry-Emery manifolds, Comm. Partial Differential Equations 37 (2012), 2081-2092.

4. D. Bakry And M. Émery, Diffusion hypercontractives, in: Séminaire de Probabilités XIX, Lect. Notes in Math. 1123, Springer, Berlin, 1985, 177-206.

5. D. BAKRY AND Z.-M. QIAN, Volume comparison theorems without Jacobi fields, in: Current Trends in Potential Theory, in: Theta Ser. Adv. Math., Theta, Bucharest, 4 (2005), 115-122. 
JIA-YONG WU

6. D. BAKRY AND Z.-M. QIAN, Some new results on eigenvectors via dimension, diameter, and Ricci curvature, Adv. in Math. 155 (2000), 98-153.

7. H.-D. CAO, Recent progress on Ricci solitons. In Recent Advances in Geometric Analysis, Advanced Lectures in Mathematics (ALM), 11, International Press, 2010, 1-38.

8. J. Case, Y.-J. Shu And G.-F. Wei, Rigidity of quasi-Einstein metrics, Diff. Geo. Appl. 29 (2011) 93-100.

9. M.-F. Chen And F.-Y. WAng, Application of coupling method to the first eigenvalue on manifolds, Sci. Sinica Ser. A 37 (1994), 1-14.

10. M.-F. Chen And F.-Y. WAng, General formula for lower bound of the first eigenvalue on Riemannian manifolds, Sci. Sinica Ser. A 40 (1997), 384-394.

11. F.-Q. FAnG, X.-D. Li and Z.-L. Zhang, Two generalizations of Cheeger-Gromoll splitting theorem via Bakry-Émery Ricci curvature, Annales de l'Institut Fourier 59 (2009), 563-573.

12. A. Futaki, H.-Z. Li And X.-D. Li, On the first eigenvalue of the Witten-Laplacian and the diameter of compact shrinking Ricci solitons, Ann. Glob. Anal. Geom. 44 (2013), 105-114.

13. A. Futaki, Y. SAno, Lower diameter bounds for compact shrinking Ricci solitons, Asian J. Math. 17 (2013), 17-32.

14. R. S. Hamilton, The formation of singularities in the Ricci flow, Surveys in Differential Geometry, 2, International Press, Boston, 1995, 7-136.

15. F.-B. Hang And X.-D. WAng, A remark on Zhong-Yang's eigenvalue estimate, Int. Math. Res. Not. 18 (2007), Art. ID rnm064, 9pp.

16. C.-X. He, P. Petersen and W. Wylie, On the classification of warped product Einstein metrics, Comm. Anal. Geom. 20 (2012), 271-312.

17. J. Jauregui and W. Wylie, Conformal diffeomorphisms of gradient Ricci solitons and generalized quasi-Einstein manifolds, J. Geom. Anal. 25 (2015), 668-708.

18. K. KuwadA, A probabilistic approach to the maximal diameter theorem, Math. Nachr. 286 (2013), 374-378.

19. S. LAKZIAN, Characterization of equality in Zhong-Yang type (sharp) spectral gap estimates for metric measure spaces, arXiv:1506.04936.

20. P. Li AND S.-T. YAU, Estimates of eigenvalues of a compact Riemannian manifold, Proc. Sympos. Pure Math. 36 (1980), 205-239.

21. X.-D. LI, Liouville theorems for symmetric diffusion operators on complete Riemannian manifolds, J. Math. Pure. Appl. 84 (2005), 1295-1361.

22. X.-D. Li, Perelman's entropy formula for the Witten Laplacian on Riemanian manifolds via Bakry-Emery Ricci curvature, Math. Ann. 353 (2012), 403-437.

23. J. Lотт, Some geometric properties of the Bakry-Émery-Ricci tensor, Comment. Math. Helv. 78 (2003), 865-883.

24. O. Munteanu And J.-P. WAng, Smooth metric measure spaces with nonnegative curvature, Comm. Anal. Geom. 19 (2011), 451-486.

25. O. Munteanu and J.-P. Wang, Analysis of weighted Laplacian and applications to Ricci solitons, Comm. Anal. Geom. 20 (2012), 55-94.

26. L. Ni, Estimates on the modulus of expansion for vector fields solving nonlinear equations, Jour. Math. Pures Appl. 99 (2013), 1-16.

27. S. Pigola, M. Rimoldi and A. G. Setti, Remarks on non-compact gradient Ricci solitons, Math. Z. 268 (2011), 777-790.

28. Y.-H. Su AND H.-C. ZhANG, Rigidity of manifolds with Bakry-Émery Ricci curvature bounded below, Geom. Dedicata 160 (2012), 321-331.

29. G.-F. Wei And W. Wylie, Comparison geometry for the Bakry-Émery Ricci tensor, J. Diff. Geom. 83 (2009), 377-405.

30. J.-Y. WU, Upper bounds on the first eigenvalue for a diffusion operator via Bakry-Émery Ricci curvature, J. Math. Anal. Appl. 361 (2010), 10-18.

31. J.-Y. WU, Upper bounds on the first eigenvalue for a diffusion operator via Bakry-Émery Ricci curvature II, Results. Math. 63 (2013), 1079-1094.

32. J.-Y. Wu, A note on the splitting theorem for the weighted measure, Ann. Glob. Anal. Geom. 43 (2013), 287-298.

33. J.-Y. WU, $L^{p}$-Liouville theorems on complete smooth metric measure spaces, Bull. Sci. Math. 138 (2014), 510-539.

34. J.-Y. Wu, Elliptic gradient estimates for a weighted heat equation and applications, Math. Z. 280 (2015), 451-468. 
35. J.-Y. WU AND P. Wu, Heat kernels on smooth metric measure spaces with nonnegative curvature, Math. Ann. 362 (2015), 717-742.

36. J.-Y. WU AND P. WU, Heat kernel on smooth metric measure spaces and applications, Math. Ann. 365 (2016), 309-344.

37. J.-Y. WU AND P. WU, On $L^{p}$-Liouville property for smooth metric measure spaces, arXiv: 1410.7305

38. J.-Q. ZhONG AND H.-C. YANG, On the estimates of the first eigenvalue of a compact Riemannian manifold, Sci. Sinica Ser. A 27 (1984), 1265-1273.

Department of Mathematics, Shanghai Maritime University, 1550 Haigang Avenue, Shanghai 201306, P. R. China

E-mail address: jywu81@yahoo.com 